\title{
Interobserver diagnostic reproducibility in advanced-stage endometrial carcinoma
}

\author{
Ho Jin Jung ${ }^{1}$, Soo Yeon Lee ${ }^{1}$, Jin Hwa Hong ${ }^{2}$, Yi Kyeong Chun ${ }^{1}$ \\ Departments of ${ }^{1}$ Pathology and ${ }^{2}$ Obstetrics and Gynecology, Korea University Guro Hospital, Korea University College of Medicine, Seoul, Korea
}

\begin{abstract}
Background: The accurate pathologic diagnosis and subtyping of high-grade endometrial carcinoma are often problematic, due to its atypical and overlapping histopathological features. Methods: Three pathologists reviewed 21 surgically resected cases of advancedstage endometrial carcinoma. The primary diagnosis was based only on hematoxylin and eosin stained slides. When a discrepancy arose, a secondary diagnosis was made by additional review of immunohistochemical $(\mathrm{IHC})$ stains. Finally, three pathologists discussed all cases and rendered a consensus diagnosis. Results: The primary diagnoses were identical in 13/21 cases (62\%). The secondary diagnosis based on the addition of $\mathrm{HC}$ results was concordant in four of eight discrepant cases. Among four cases with discrepancies occurring in this step, two cases subsequently reached a consensus diagnosis after a thorough discussion between three reviewers. Next-generation sequencing (NGS) study was performed in two cases in which it was difficult to distinguish between serous carcinoma and endometrioid carcinoma. Based on the sequencing results, a final diagnosis of serous carcinoma was rendered. The overall kappa for concordance between the original and consensus diagnosis was 0.566 (moderate agreement). Conclusions: We investigated stepwise changes in interobserver diagnostic reproducibility in advanced-stage endometrial carcinoma. We demonstrated the utility of IHC and NGS study results in the histopathological diagnosis of advanced-stage endometrial carcinoma.
\end{abstract}

Key Words: Endometrial neoplasms; Uterine neoplasm; Observer variation

Received: August 10, 2020 Revised: September 16, 2020 Accepted: October 4, 2020

Corresponding Author: Yi Kyeong Chun, MD, Department of Pathology, Korea University Guro Hospital, Korea University College of Medicine, 148 Gurodong-ro, Guro-gu, Seoul 08308, Korea

Tel: +82-2-2626-1472, Fax: +82-2-2626-1486, E-mail: ykcmd@naver.com

Endometrial carcinoma is the most common gynecological cancer in the Western world [1]. The annual incidence of endometrial cancer in South Korea has increased from 619 in 1999 to 2,263 in 2015 [2].

According to the 2014 WHO Classification of Tumors of Female Reproductive Organs [3], various histological subtypes of endometrial carcinoma include endometrioid carcinoma, serous carcinoma, clear cell carcinoma, carcinosarcoma, and undifferentiated/dedifferentiated carcinoma, among others. All subtypes except low-grade endometrioid carcinoma are high-grade malignancies, and are clinically more aggressive and exhibit poor prognoses $[4,5]$. Precise histological diagnosis of high-grade endometrial carcinoma can be challenging if histopathologic and immunohistochemical (IHC) characteristics are overlapping or atypical [6,7].

Several researchers have reported the utility of biomarker pan- els and molecular profiling in endometrial carcinoma diagnosis. Han et al. [8] demonstrated that a seven-marker immunostaining panel (consisting of estrogen receptor [ER], progesterone receptor $[\mathrm{PR}], \mathrm{p} 16, \mathrm{p} 53$, vimentin, PTEN, and IGF2BP3) could differentiate high-grade endometrioid carcinoma and serous carcinoma with $100 \%$ concordance. Nastic et al. [9] reported an increase in interobserver agreement rates after application of ER, PR, and p53 immunostaining and DNA ploidy studies. Molecular profiling can also aid in the differential diagnosis of endometrial carcinoma. For example, TP53 mutations and CCNE1 locus amplification are common in serous carcinoma, but rare in endometrioid carcinoma [10]. In contrast, most endometrioid carcinomas harbor ARID1A mutations, whereas serous carcinomas do not $[10,11]$.

This study was designed to assess interobserver reproducibility in the subtype diagnosis of cases previously diagnosed as ad- 
vanced-stage endometrial carcinoma in routine surgical pathology practice, under the assumption that there are more high-grade than low-grade carcinomas in cases diagnosed as advanced-stage endometrial carcinoma. Three pathologists with variable diagnostic experience reviewed the hematoxylin and eosin (H\&E)staining and IHC-stained slides. Through this study, we aimed to assess the diverse distribution of histological subtypes in advanced-stage endometrial carcinomas and evaluate the reproducibility of histological diagnosis among pathologists. We also investigated the utility and limitations of IHC staining and nextgeneration sequencing (NGS) analysis in the differential diagnosis of a few discordant cases and attempted to identify histological and IHC staining features that are useful in daily pathology practice.

\section{MATERIALS AND METHODS}

\section{Patients and cases}

Twenty-one patients with high International Federation of Gynecology and Obstetrics (FIGO) stage (stage III or IV) endometrial carcinoma who had undergone total hysterectomy in Korea University Guro Hospital (KUGH) between 2008 and 2017 were included in this study. A number of general pathologists performed the original diagnosis. Three pathologists (Y.K.C., S.Y.L., and H.J.J.) reviewed the cases; Y.K.C. is a gynecology specialty pathologist, S.Y.L. is a board-certified general pathologist, and H.J.J. is a fourth-year anatomic pathology resident.

\section{Three-step assessment of interobserver reproducibility}

The diagnostic process proceeded as follows. First, three reviewers reached a primary diagnosis based on the H\&E-stained slides. Each case was diagnosed based on the 2014 WHO Classification of Tumors of Female Reproductive Organs [3], and was categorized into one of the following entities: endometrioid carcinoma, serous carcinoma, mucinous carcinoma, clear cell carcinoma, undifferentiated/dedifferentiated carcinoma, carcinosarcoma, and mixed carcinoma. Cases diagnosed as endometrioid carcinoma were classified as low-grade (FIGO grade 1 and 2) or high-grade (FIGO grade 3).

In cases with discrepant original and primary diagnoses, reviewers reached a secondary diagnosis based on the H\&E-stained and IHC-stained slides. Finally, all three pathologists conducted a discussion and reached a consensus diagnosis. If the discussion did not lead to a consensus diagnosis, NGS analysis was performed.

Concordance between the original and consensus diagnoses was calculated using the kappa statistics. A kappa value of 0.4 indicates poor agreement, $0.4-0.6$ indicates moderate agreement, 0.6-0.8 indicates substantial/good agreement, and 0.81.0 indicates near perfect/ excellent agreement. The Windows version of IBM SPSS ver. 24.0 (IBM Corp., Armonk, NY, USA) was used for statistical analysis.

\section{IHC staining and interpretation}

IHC staining was performed on whole slide sections using two automated staining systems, including a Bond-III autostainer (Leica Biosystems, Wetzlar, Germany) and a Bench-Mark ULTRA system (Ventana Medical Systems, Tucson, AZ, USA). In brief, 4- $\mu$ m-thick paraffin-embedded tissue sections were deparaffinized and rehydrated across a graded series of ethyl alcohol concentrations. Heat-induced antigen retrieval was carried out in citrate buffer. Sections were incubated with the primary antibody in an automated immunostainer. Counterstaining with hematoxylin was performed. The antibodies used were as follows: anti-p53 (1:200, DO-7, Novocastra, Newcastle, UK), anti-ER (Ready-To-Use [RTU], SP1, Roche, Basel, Switzerland), anti-PR (RTU, 1E2, Roche), anti-p16 (RTU, E6H4, Roche), anti-MLH1 (1:100, ES05, Novocastra), anti-MSH2 (1:400, G219-1129, Novocastra), anti-PMS2 (1:100, MRQ-28, Cell Marque, Rocklin, CA, USA), anti-MSH6 (1:200, 44, Cell Marque), anti-hepatocyte nuclear factor-1 $\beta$ (HNF-1 $\beta ; 1: 200$, polyclonal, Sigma-Aldrich, St. Louis, MO, USA), anti-napsin A (1:100, polyclonal, Cell Marque), anti-CD56 (1:200, 123 C3, DAKO, Glostrup, Denmark), anti-synaptophysin (1:200, polyclonal, Cell Marque,), anti-chromogranin (1:500, Dak-A3, DAKO), anti-GATA3 (1:50, L50-823, Cell Marque), anti-CD10 (RTU, CC1, Roche), anti-thyroid transcription factor-1 (TTF-1; 1:200, 8G7G3/1, DAKO), anti-WT1 (1:200, 6F-H2, Cell Marque), anti-cytokeratin (CK; 1:200, AE1/AE3, DAKO), anti-vimentin (1:1000, Vim 384, DAKO), anti-Ki67 (1:100, MIB-1, DAKO), anti-PTEN (1:400, 6H2-1, DAKO), and anti-c-KIT (1:200, polyclonal, Novocastra).

Each reviewer independently assessed the results of IHC staining. When interpretation-associated discrepancies arose, all three reviewers conducted a discussion until a consensus was achieved. p53 immunostaining was interpreted as mutation type if the tumor exhibited: (1) diffuse strong nuclear positivity involving at least $80 \%$ of the tumor cells (aberrant pattern), (2) complete absence of staining with the presence of positive internal control staining of non-neoplastic cells such as lymphocytes (null pattern), or (3) unequivocal cytoplasmic staining accompanied by variable nuclear staining (cytoplasmic staining pattern). Cases were considered wild-type if any degree of non-diffuse nucle- 
ar staining $(<80 \%)$ of the tumor cells was present [12]. Immunostaining for ER and PR was scored based on the percentage of tumor cells exhibiting moderate to intense nuclear staining. p16 staining was interpreted as positive if $\geq 90 \%$ of tumor cells were stained. PTEN immunostaining was considered negative if there was a complete loss of expression in tumor cells. Immunostaining for MLH1, MSH2, MSH6, and PMS2 was considered negative (loss of expression pattern) if there was a complete absence of nuclear staining. Immunostaining for napsin $A$ and WT1 was interpreted as positive if $\geq 1 \%$ of the tumor cells were stained, regardless of the staining intensity. HNF-1 $\beta$ immunostaining was interpreted as positive if there was positive staining in $\geq 50 \%$ of tumor cells. Immunostaining for neuroendocrine markers (CD56, synaptophysin, and chromogranin) was interpreted as positive if there was positive staining in $\geq 10 \%$ of tumor cells. Immunostaining for CK and vimentin was considered positive if there was diffuse staining in $\geq 50 \%$ of tumor cells. Immunostaining for CD10, GATA3, and TTF-1 was interpreted as positive if there was more than one area of focal staining. c-KIT immunostaining was considered positive if there was cytoplasmic staining in $\geq 1 \%$ of tumor cells. The Ki67 labeling index was measured by recording the tumor cell percentages that exhibited moderate to intense nuclear staining.

\section{Next-generation sequencing}

Up to five 10- $\mu$ m-thick sections of formalin-fixed, paraffinembedded tissue blocks were used for DNA and RNA sequencing analysis. Representative tumor areas were manually microdissected. DNA and RNA were extracted using the Invitrogen RecoverAll Total Nucleic Acid Isolation Kit (Invitrogen, Carlsbad, CA, USA). Massively parallel sequencing of cancer-related gene panels (Oncomine Comprehensive Assay v3, ThermoFisher Scientific Waltham, MA, USA) was performed using the Ion Chef and Ion Torrent sequencing platforms. Sequence data were aligned against the human reference genome (hg19 build). Bioinformatics data analysis was performed using Ion Reporter software (v.5.10.1.0) with the Oncomine Variants filter chain (v.5.6).

\section{RESULTS}

\section{Interobserver diagnostic reproducibility}

The original diagnoses in 21 cases were endometrioid carcinoma $(n=15)$, serous carcinoma $(n=5)$, and clear cell carcinoma ( $=1$ ). Among the 15 cases of endometrioid carcinoma, five were low grade (G1, 2 cases; G2, three cases), and 10 cases were high grade $(\mathrm{G} 3)$. The primary diagnoses made by the three reviewers were identical in 13/21 cases (62\%). These cases included endometrioid carcinoma $(n=9)$, serous carcinoma $(n=3)$, and clear cell carcinoma $(n=1)$. Table 1 showed a summary of the primary diagnosis.

Secondary diagnosis due to disagreement in primary diagnosis or diagnostic difficulty was performed in 8/21 cases (38\%). Table 2 summarized the IHC staining result in eight discordant cases. The original diagnosis of eight cases with discrepancies was endometrioid carcinoma in six cases (one case of G1 and five cases of G3) and serous carcinoma in two cases. Secondary diagnosis based on H\&E and IHC staining was concordant in four of eight discrepant cases. The secondary diagnosis of four concordant cases was mesonephric-like adenocarcinoma (case 1), carcinosarcoma (case 2), clear cell carcinoma (case 3), and large cell neuroendocrine carcinoma (LCNEC) (case 4). The four discordant cases were as follows: mixed carcinoma vs. LCNEC (case 5), G3 endometrioid vs. dedifferentiated carcinoma (case 6), and G3 endometrioid vs. serous carcinoma (cases 7 and 8).

Among four discordant cases, two cases reached a consensus diagnosis through review of all slides and discussion between three

Table 1. Original diagnosis and primary diagnosis of all cases

\begin{tabular}{|c|c|c|c|c|}
\hline \multirow{2}{*}{$\begin{array}{l}\text { Case } \\
\text { No. }\end{array}$} & \multirow{2}{*}{$\begin{array}{c}\text { Original } \\
\text { diagnosis }\end{array}$} & \multicolumn{3}{|c|}{ Primary diagnosis } \\
\hline & & Reviewer 1 & Reviewer 2 & Reviewer 3 \\
\hline 1 & G1 EC & MesL & Low-grade EC & Low-grade EC \\
\hline 2 & SC & SC vs. CS & SC & SC \\
\hline 3 & G3 EC & G3 EC & $\mathrm{CC}$ & G3 EC \\
\hline 4 & G3 EC & LCNEC & G3 EC & LCNEC \\
\hline 5 & G3 EC & $\begin{array}{c}\text { Mixed (G3 EC+ } \\
\text { CC+LCNEC) }\end{array}$ & G3 EC & SC \\
\hline 6 & G3 EC & $\mathrm{DD}$ & $\mathrm{DD}$ & SC \\
\hline 7 & G3 EC & G3 EC vs. SC & SC & G3 EC \\
\hline 8 & SC & G3 EC vs. SC & SC & SC \\
\hline 9 & G1 EC & Low-grade EC & Low-grade EC & Low-grade EC \\
\hline 10 & G2 EC & Low-grade EC & Low-grade EC & Low-grade EC \\
\hline 11 & G2 EC & Low-grade EC & Low-grade EC & Low-grade EC \\
\hline 12 & G2 EC & Low-grade EC & Low-grade EC & Low-grade EC \\
\hline 13 & G3 EC & G3 EC & G3 EC & G3 EC \\
\hline 14 & G3 EC & G3 EC & G3 EC & G3 EC \\
\hline 15 & G3 EC & G3 EC & G3 EC & G3 EC \\
\hline 16 & G3 EC & G3 EC & G3 EC & G3 EC \\
\hline 17 & G3 EC & G3 EC & G3 EC & G3 EC \\
\hline 18 & SC & SC & SC & SC \\
\hline 19 & SC & SC & SC & SC \\
\hline 20 & SC & SC & SC & SC \\
\hline 21 & $\mathrm{CC}$ & $\mathrm{CC}$ & $\mathrm{CC}$ & $\mathrm{CC}$ \\
\hline
\end{tabular}

G1, grade 1; G2, grade 2; G3, grade 3; EC, endometrioid carcinoma; MesL, mesonephric-like adenocarcinoma; SC, serous carcinoma; CS, carcinosarcoma; CC, clear cell carcinoma; LCNEC, large cell neuroendocrine carcinoma; Mixed, mixed cell adenocarcinoma; DD, dedifferentiated carcinoma. 
pathologists. The consensus diagnosis was LCNEC (case 5) and dedifferentiated carcinoma (case 6). In two cases of $\mathrm{G} 3$ endometrioid vs. serous carcinoma (cases 7 and 8), NGS was performed because a consensus was not achieved even after discussion. Table 3 was a sequential diagnosis summary of eight discordant cases.

Through the above diagnostic process, three reviewers made a consensus diagnoses for 21 advanced-stage endometrial carcinoma cases. The final diagnoses included four cases of low-grade endometrioid carcinoma, five cases of high-grade (G3) endometrioid carcinoma, five cases of serous carcinoma, two cases of clear cell carcinoma, two cases of neuroendocrine carcinoma, one case of mesonephric-like adenocarcinoma, one case of carcinosarcoma, and one case of dedifferentiated carcinoma (Table 4). The overall kappa for concordance between the original diagnosis and consensus diagnosis was 0.566 (moderate agreement).

\section{Cases with a consensus diagnosis from $\mathrm{IHC}$ staining}

The original diagnosis of case 1 was G1 endometrioid carcinoma. Three pathologists' primary diagnosis were discordant, as one pathologist diagnosed this case as mesonephric-like adenocarcinoma and two others diagnosed it as low-grade endometrioid carcinoma. The tumor exhibited diverse histological morphology such as papillary and ductal/glandular patterns with a low nuclear grade. Nuclear features of the cells included nuclear overlapping, nuclear grooves, and open, vesicular chromatin, similar to those of papillary thyroid carcinoma. Although the histopathological characteristics were consistent with those of mesonephric adenocarcinoma, mesonephric remnants and uterine cervical involvement were not identified. IHC staining showed positivity in GATA3 and TTF-1, negativity in CD10 and ER, and focal positivity in PR. Based on pathologic findings and IHC results, the consensus diagnosis was mesonephric-like adenocarcinoma (Fig. 1A, B).

In case 2 , the original diagnosis was serous carcinoma. This primary diagnosis was discordant as two pathologists diagnosed this as serous carcinoma, while the remaining pathologist could not decide between serous carcinoma and carcinosarcoma. Microscopic findings showed serous and mesonephric carcinoma

Table 2. Immunohistochemical staining results of eight discordant cases

\begin{tabular}{cccccl}
\hline Case No. & Original diagnosis & p53 & ER & PR & \multicolumn{1}{c}{ Others } \\
\hline 1 & G1 EC & Wild type & $(-)$ & $(+), 30 \%$ & GATA3 (+), TTF-1 (+), CD10 (-) \\
2 & SC & Aberrant & $(+), 10 \%$ & $(+), 5 \%$ & $\begin{array}{l}\text { p16 (+, diffuse in carcinoma) } \\
\text { CK (+, diffuse in carcinoma, focal in sarcoma) } \\
\end{array}$ \\
& & & & $\begin{array}{l}\text { Vimentin (+ in sarcoma) } \\
\text { WT1 (+ in carcinoma) }\end{array}$ \\
& & & & Napsin A (+), HNF-1 $(-)$ \\
3 & G3 EC & Wild type & $(-)$ & $(-)$ & CD56 (+), chromogranin (+), synaptophysin (-) \\
5 & G3 EC & Wild type & $(+), 30 \%$ & $(-)$ & CD56 (+), synaptophysin (+), chromogranin $(+$, focal) \\
6 & G3 EC & Wild type & $(+), 20 \%$ & $(+), 15 \%$ & PTEN (+), WT1 (-) \\
7 & G3 EC & Wild type & $(+), 10 \%$ & $(+), 5 \%$ & pMMR \\
8 & G3 EC & Null & $(-)$ & $(-)$ & p16 (+, diffuse), pMMR, WT1 (+), PTEN (+) \\
\hline
\end{tabular}

ER; estrogen receptor; PR, progesterone receptor; EC, endometrioid carcinoma; G1, grade 1; G3, grade 3; TTF-1, thyroid transcription factor-1; SC, serous carcinoma; CK, cytokeratin; pMMR, proficient mismatch repair.

Table 3. Sequential diagnosis of discordant cases

\begin{tabular}{|c|c|c|c|c|c|c|c|c|}
\hline \multirow{2}{*}{ Case No. } & \multirow{2}{*}{$\begin{array}{c}\text { Original } \\
\text { diagnosis }\end{array}$} & \multicolumn{3}{|c|}{ Primary diagnosis } & \multicolumn{3}{|c|}{ 2nd diagnosis } & \multirow{2}{*}{$\begin{array}{c}\text { Consensus } \\
\text { diagnosis }\end{array}$} \\
\hline & & Reviewer 1 & Reviewer 2 & Reviewer 3 & Reviewer 1 & Reviewer 2 & Reviewer 3 & \\
\hline 1 & G1 EC & MesL & Low-grade EC & Low-grade EC & MesL & MesL & MesL & MesL \\
\hline 2 & SC & SC vs. CS & SC & SC & CS & CS & CS & CS \\
\hline 3 & G3 EC & G3 EC & $\mathrm{CC}$ & G3 EC & $\mathrm{CC}$ & $\mathrm{CC}$ & $\mathrm{CC}$ & $\mathrm{CC}$ \\
\hline 4 & G3 EC & LCNEC & G3 EC & LCNEC & LCNEC & LCNEC & LCNEC & LCNEC \\
\hline 5 & G3 EC & $\begin{array}{c}\text { Mixed (G3 EC+ } \\
\text { CC + LCNEC) }\end{array}$ & G3 EC & SC & LCNEC & LCNEC & $\begin{array}{l}\text { Mixed (low-grade } \\
\text { EC + LCNEC) }\end{array}$ & LCNEC \\
\hline 6 & G3 EC & $\mathrm{DD}$ & $\mathrm{DD}$ & SC & $\mathrm{DD}$ & $\mathrm{DD}$ & G3 EC & $\mathrm{DD}$ \\
\hline 7 & G3 EC & G3 EC vs. SC & SC & G3 EC & G3 EC vs. SC & SC & SC & $\mathrm{SC}^{\mathrm{a}}$ \\
\hline 8 & SC & SC vs. G3 EC & SC & SC & G3 EC & SC & G3 EC & $\mathrm{SC}^{\mathrm{a}}$ \\
\hline
\end{tabular}

G1, grade 1; G2, grade 2; G3, grade 3; EC, endometrioid carcinoma; MesL, mesonephric-like adenocarcinoma; SC, serous carcinoma; CS, carcinosarcoma; LCNEC, large cell neuroendocrine carcinoma; Mixed, mixed cell adenocarcinoma; CC, clear cell carcinoma; DD, dedifferentiated carcinoma.

${ }^{a}$ Consensus diagnosis was made after next-generation sequencing analysis. 
Table 4. Original diagnosis and consensus diagnosis of all reviewed cases

\begin{tabular}{ccc}
\hline Case No. & Original diagnosis & Consensus diagnosis \\
\hline 1 & G1 EC & MesL \\
2 & SC & CS \\
3 & G3 EC & CC \\
4 & G3 EC & LCNEC \\
5 & G3 EC & LCNEC \\
6 & G3 EC & DD \\
7 & G3 EC & SC \\
8 & SC & SC \\
9 & G1 EC & Low-grade EC \\
10 & G2 EC & Low-grade EC \\
11 & G2 EC & Low-grade EC \\
12 & G2 EC & Low-grade EC \\
13 & G3 EC & G3 EC \\
14 & G3 EC & G3 EC \\
15 & G3 EC & G3 EC \\
16 & G3 EC & G3 EC \\
17 & G3 EC & G3 EC \\
18 & SC & SC \\
19 & SC & SC \\
20 & SC & SC \\
21 & GC & CC \\
\hline
\end{tabular}

G1, grade 1; G2, grade 2; G3, grade 3; EC, endometrioid carcinoma; MesL, mesonephric-like adenocarcinoma; SC, serous carcinoma; CS, carcinosarcoma; CC, clear cell carcinoma; LCNEC, large cell neuroendocrine carcinoma; DD, dedifferentiated carcinoma.

components. Two small foci of atypical spindle and pleomorphic cell proliferation suggested the presence of a sarcomatous component. The spindle cell component exhibited strong vimentin positivity and aberrant $\mathrm{p} 53$ expression, while $\mathrm{CK}$ expression was focal and weak. After discussion, the three pathologists agreed upon the presence of a distinct sarcomatous component, and a consensus diagnosis of carcinosarcoma was made (Fig. 1C, D).

Case 3 showed solid or glandular proliferation patterns, and the tumor cells displayed severe nuclear atypia and abundant clear or eosinophilic cytoplasm. The primary diagnosis was discordant, with one diagnosis of clear cell carcinoma and two diagnoses of endometrioid carcinoma. On IHC stains, the tumor cells were $\mathrm{p} 53$ wild-type and negative for ER, PR, and HNF-1 $\beta$ with granular positivity for napsin $\mathrm{A}$. The final consensus diagnosis was clear cell carcinoma (Fig. 1E, F).

In case 4, large polygonal cells with prominent nucleoli and abundant cytoplasm formed well-demarcated nests, which displayed peripheral palisading. Frequent mitotic figures were observed, and geographic tumor necrosis was identified. The primary diagnosis was discordant, with two diagnoses of neuroendocrine carcinoma and one diagnosis of $\mathrm{G} 3$ endometrioid carcinoma. The tumor cells revealed diffuse positivity to $\mathrm{CD} 56$ and chromogranin.
A consensus diagnosis of LCNEC was rendered (Fig. 1G, H).

\section{Cases confirmed by consensus discussion}

For case 5, the primary diagnoses were mixed carcinoma (endometrioid carcinoma, clear cell carcinoma, and neuroendocrine carcinoma) vs. G3 endometrioid carcinoma vs. serous carcinoma. Histopathologic analysis showed that the tumor was composed of endometrioid carcinoma (50\%), clear cell carcinoma (30\%), and LCNEC (20\%). The IHC staining revealed an LCNEC component showing diffuse positivity for $\mathrm{CD} 56$ and synaptophysin and focal positivity for chromogranin. Although the tumor consisted of various components, according to the criteria of the WHO Classification of Tumors of Female Reproductive Organs [3], case 5 was diagnosed as LCNEC (Fig. 2A, B).

In case 6 , the primary diagnosis was divided into two diagnoses of dedifferentiated carcinoma and one diagnosis of serous carcinoma. The secondary diagnosis was also discordant, as two pathologists diagnosed the case as dedifferentiated carcinoma and the remaining pathologist's diagnosis was G3 endometrioid carcinoma. Microscopically, the tumor was composed of G1 endometrioid carcinoma and a poorly differentiated solid portion showing non-cohesive cells without readily visible gland formation. The three reviewers agreed that the solid portion was an undifferentiated carcinoma, not a high-grade endometrioid carcinoma component. A final consensus diagnosis of dedifferentiated carcinoma was reached (Fig. 2C, D).

\section{Cases that reached consensus diagnosis with NGS}

The microscopic features of case 7 displayed columnar tumor cells with nuclear pseudostratification and pleomorphism. While the tumor's dominant pattern was solid proliferation, several foci showed complex papillary or glandular patterns. IHC staining revealed a complete loss of $\mathrm{p} 53$ staining, negativity for ER and PR, and positivity for p16, WT1, MLH1, PMS2, MSH2, and MSH6. NGS analysis was performed as histopathological features (suggestive of endometrioid carcinoma) and IHC features (suggestive of serous carcinoma) exhibited discrepancies (Fig. 2E, F). NGS revealed mutations in TP53, PIK3CA, and NF1, and copy number variations (CNVs) in MYCL, FGFR3, CDK2, $C D K 4, E R B B 2$, and CCNE1. As the molecular profile corresponded to a copy-number high group, the final diagnosis was rendered as serous carcinoma.

Case 8 presented with high-grade tumor cells with severe nuclear pleomorphism. The tumor showed complex glandular and solid patterns. In the solid portion, nuclear pleomorphism was less severe than that of typical serous carcinoma. The primary 

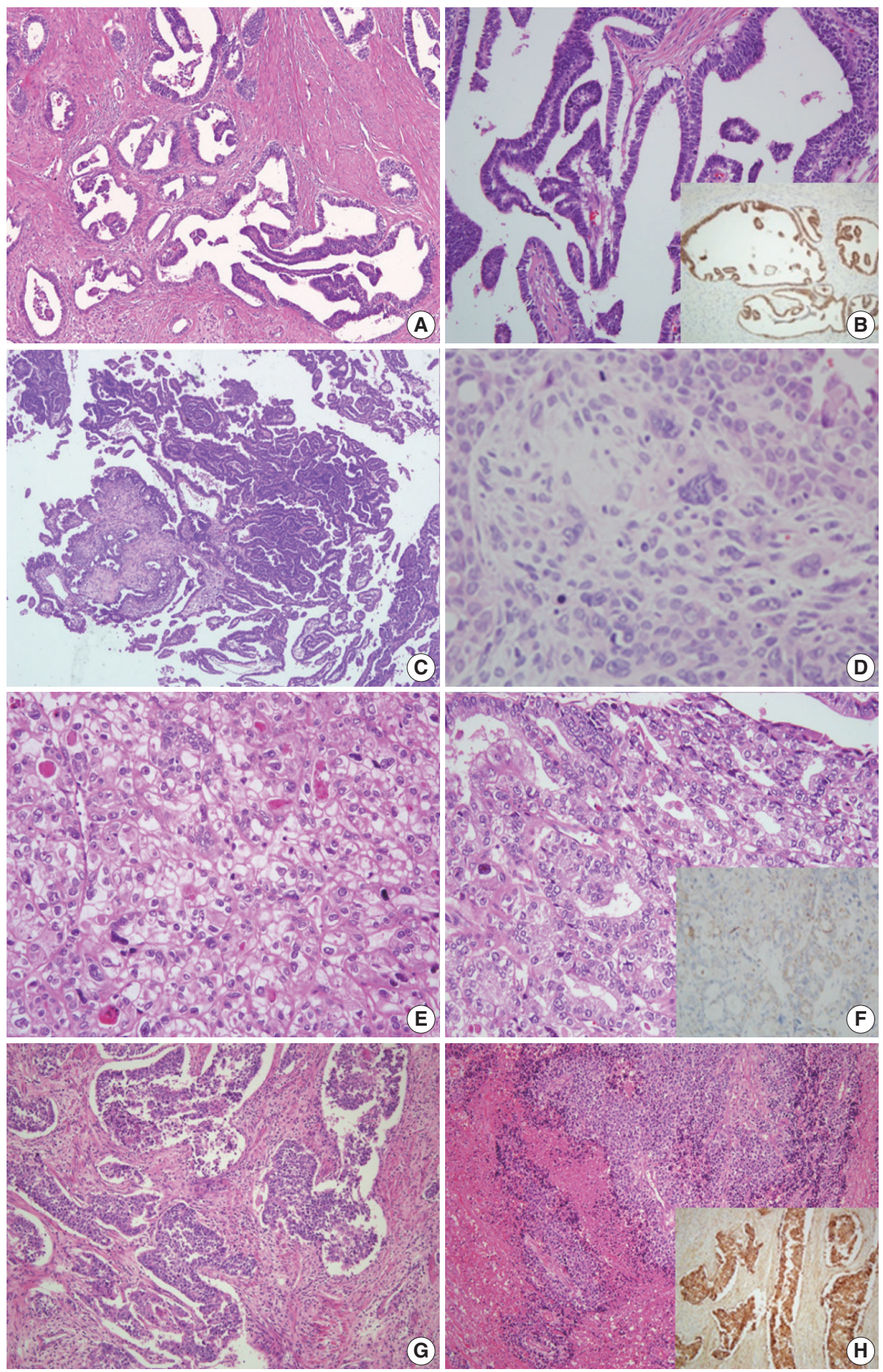

Fig. 1. Representative microscopic features of cases 1-4. (A) In case 1 (mesonephric-like adenocarcinoma), the tumor showed ductal/glandular and papillary patterns. (B) Nuclear features of tumor cells were similar to those of papillary thyroid carcinoma, showing nuclear overlapping and openness of vesicular chromatin. Immunohistochemical (IHC) staining showed positivity in GATA3 (inset). (C) In case 2 (carcinosarcoma), the tumor predominantly showed complex papillary components and small foci of hypercellular stromal lesions. (D) On higher magnification, this lesion consisted of atypical pleomorphic spindle cells with frequent mitosis. (E) In case 3 (clear cell carcinoma), the tumor mainly showed a solid pattern. The tumor cells had hyperchromatic nuclei with vesicular chromatin and eosinophilic or clear cytoplasm. (F) A portion of the tumor showed a glandular pattern with high-grade nuclear atypia. On IHC stains, the tumor cells exhibited granular positivity for napsin A (inset). (G) In case 4 (large cell neuroendocrine carcinoma), the tumor showed well-defined nests with peripheral palisading. $(H)$ Geographic tumor necrosis and diffuse immunoreactivity to CD56 was seen (inset). 

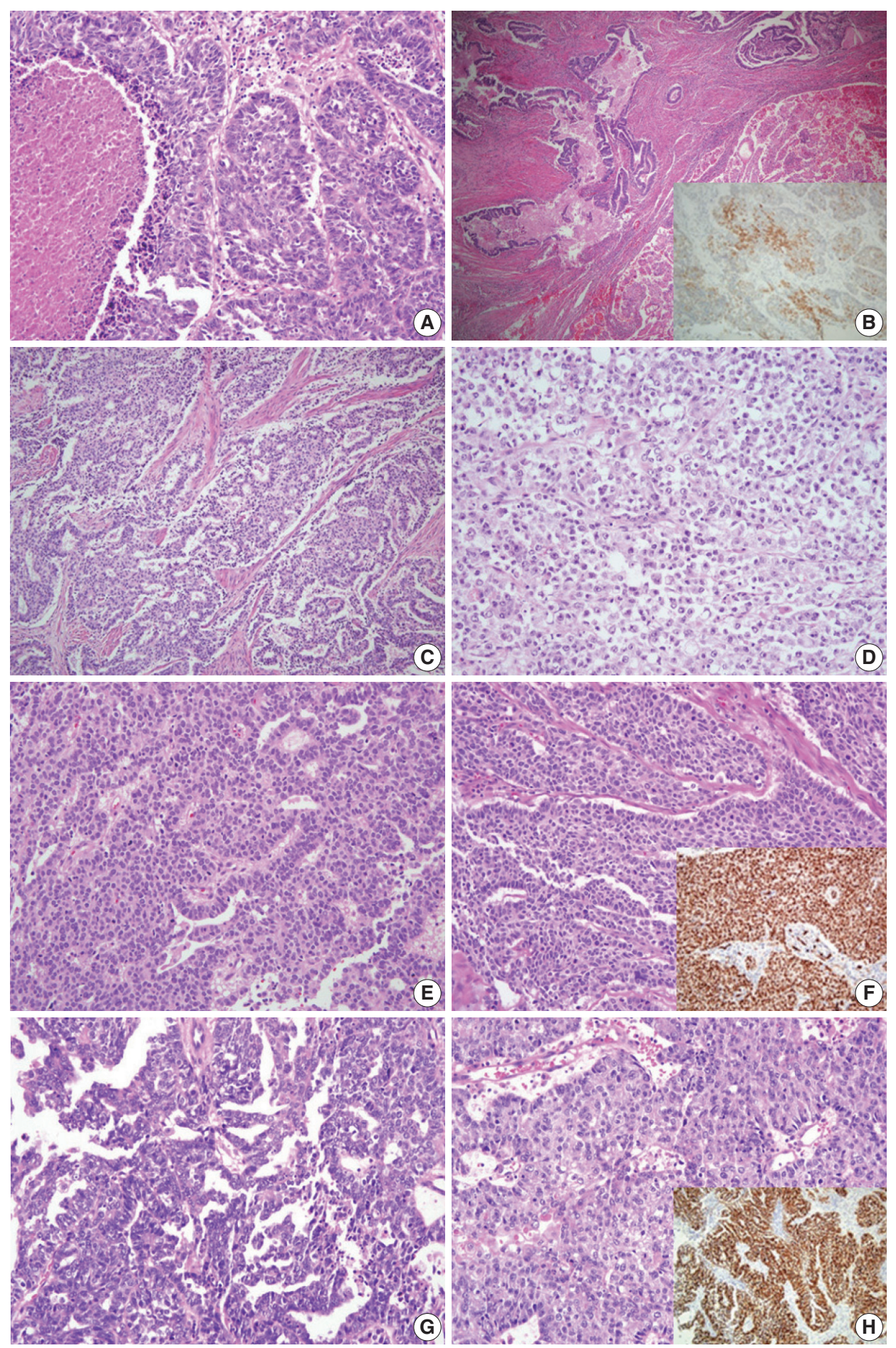

Fig. 2. Representative microscopic features of cases 5-8. (A) In case 5 (large cell neuroendocrine carcinoma), a portion of the tumor was composed of well-defined nests with peripheral palisading and geographic necrosis. (B) The tumor also partly showed complex glandular and papillary pattern. The Immunohistochemical $(\mathrm{IHC})$ staining of tumor cells showed diffuse positivity for synaptophysin (inset). (C) In case 6 (dedifferentiated carcinoma), the tumor showed complex glandular architecture consisting of columnar cells. (D) The undifferentiated carcinoma component was composed of solid sheets of monotonous dyscohesive cells. (E) In case 7 (serous carcinoma), the tumor showed a focal complex glandular and papillary pattern. (F) It was mostly comprised of solid architecture with high-grade nuclei and diffuse positivity to WT1 (inset). (G) In case 8 (serous carcinoma), some areas of the tumor showed papillary and micropapillary architecture composed of columnar cells with prominent nucleoli and nuclear pleomorphism. $(\mathrm{H})$ Most of the tumor showed a solid pattern with focal glandular differentiation with tumor cells showing mild nuclear atypia. On immunostaining, the tumor cells exhibited aberrant p53 expression (inset). 
diagnoses were divided into $G 3$ endometrioid carcinoma versus serous carcinoma. On immunostaining, the tumor cells exhibited aberrant p53 expression, negativity for PR, diffuse positivity for p16, MLH1, PMS2, MSH2, and MSH6, and focal positivity for WT1 and ER. While the immunoprofile suggested serous carcinoma, the diagnosis of G3 endometrioid carcinoma could not be entirely excluded for the histomorphological findings (Fig. 2G, H). NGS analysis revealed mutations in TP53 and polymerase $\mathrm{E}$ (POLE) and CNVs involving ALK, ERBB2, CCNE1, $A K T 2$, and AXL. Although POLE mutation was present, the total tumor mutation burden was not as high as that seen in the typical POLE-ultramutated type. Since a significant number of CNVs were detected, the case was assigned to the copy-number high group, and a final diagnosis of serous carcinoma was made.

\section{DISCUSSION}

Diagnosis of high-grade endometrial carcinoma has been shown to be unreproducible, even among gynecologic pathology specialists. In several studies involving high-grade endometrial carcinoma, the consensus rate of cancer histotypes among the participating pathologists varied from $39 \%$ to $72 \%[7,8,13]$. According to Gilks et al. [7], a diagnostic consensus among three reviewers about the exclusive or major subtype of high-grade endometrial carcinoma was reached in only $62.5 \%$ of cases. We studied diagnostic agreement in high-grade endometrial carcinoma cases to evaluate interobserver reproducibility, based on the assumption that advanced-stage endometrial carcinoma would predominantly consist of high-grade endometrial carcinomas.

Of the advanced-stage endometrial carcinomas included in this study, the original diagnosis and the primary diagnosis by three reviewers were identical in 13/21 cases (62\%). These cases had typical histopathological features, and the diagnoses included nine cases of endometrioid carcinoma, three cases of serous carcinoma, and one case of clear cell carcinoma. Among the eight cases involving discordant primary diagnoses, six cases were initially diagnosed as endometrioid carcinoma and two cases was diagnosed as serous carcinoma. We attained diagnostic agreement for six of the eight cases based on characteristic histopathological features and IHC staining results. The cases in which meticulous histologic examination alone led to an agreed diagnosis were carcinosarcoma and dedifferentiated carcinoma. The diagnosis of LCNEC, mesonephric-like adenocarcinoma, and clear cell carcinoma were achieved by panel of IHC staining. The overall kappa for concordance between the original and consensus diagnosis was 0.566 (moderate agreement). Other studies about interobserver reproducibility also show similar kappa values $[8,13]$.

In cases 7 and 8, it was difficult to differentiate between serous carcinoma and G3 endometrioid carcinoma. Both cases exhibited papillary growth and marked nuclear atypia, and immunostaining findings suggested a diagnosis of serous carcinoma. However, the possibility of $\mathrm{G} 3$ endometrioid carcinoma could not be wholly excluded. Molecular profiling by NGS was performed to reach a final diagnosis. In a comprehensive genomic analysis of endometrioid and serous carcinoma based on the The Cancer Genome Atlas database, endometrioid and serous carcinomas were divided into four molecular subgroups [11], including POLE ultramutated, microsatellite instability-hypermutated, copynumber low, and copy-number high; serous carcinoma belongs only to the copy-number high group. Endometrioid carcinoma belongs to all four groups [14]. According to this molecular classification scheme, cases 7 and 8 were designated as copy-number high group and finally diagnosed as serous carcinoma.

In daily clinical practice settings, where molecular studies cannot be performed routinely, histopathological examination and IHC panel staining help to differentiate $\mathrm{G} 3$ endometrioid carcinoma from serous carcinoma. If the tumor shows papillary growth, cellular tufts, and diffuse high-grade nuclear atypia, a diagnosis of serous carcinoma is favored. When defining endometrioid features such as squamous or mucinous differentiation on a background of endometrial hyperplasia present, a diagnosis of endometrioid carcinoma can be rendered. The use of a panel of IHC stains can help to establish a confirmatory diagnosis when histomorphological findings are unclear or overlapping $[6,9,15]$. Biomarkers commonly used to distinguish between endometrioid and serous carcinomas are immunostaining for p53, p16, ER, PR, PTEN, and DNA mismatch repair proteins $[6,9,12,16,17]$. Tumors lacking aberrant $\mathrm{p} 53$ expression are unlikely to be serous carcinoma [17].

The final diagnosis of case 1 was mesonephric-like adenocarcinoma, whereas the original diagnosis was G1 endometrioid carcinoma. Mesonephric carcinoma exhibits diverse morphology, such as tubular, glandular, papillary, retiform, and glomeruloid patterns, and nuclei resembling those of papillary thyroid carcinoma, with dense focal eosinophilic intraluminal secretions $[17,18]$. Mesonephric-like adenocarcinoma can be differentiated from low-grade endometrioid carcinoma by immunostaining for ER and PR, as mesonephric-like adenocarcinoma rarely shows immunoreactivity to these antigens. As mesonephric-like adenocarcinoma shows aggressive clinical behavior a with preponderance for pulmonary metastasis [18], accurate diagnosis of this 
tumor is of critical importance.

Endometrial neuroendocrine carcinoma is a rare, highly aggressive tumor, and has a propensity for systemic spread and poor prognosis [19]. In this study, two LCNEC cases were originally diagnosed as G3 endometrioid carcinoma. Neuroendocrine carcinoma usually presents as pure neuroendocrine carcinoma or a mixture of other epithelial neoplasias, most commonly endometrioid carcinoma. The diagnosis of endometrial neuroendocrine carcinomas is challenging, due to its frequent association with low-grade endometrioid carcinoma. Awareness of these facts may help to avoid overdiagnosis or misdiagnosis of neuroendocrine carcinoma, especially in quantitatively limited samples [19].

The dedifferentiated carcinoma identified in this study (case 6) was composed of low-grade endometrioid carcinoma and undifferentiated carcinoma. Undifferentiated carcinoma is defined as a malignant epithelial neoplasm composed of small to intermediate-sized cells arranged in sheets, without any apparent glandular differentiation; it often exhibits a characteristically 'dyscohesive' pattern $[6,17]$. When combined with low-grade endometrioid carcinoma, it is often misinterpreted as a solid growth of endometrioid carcinoma. The original diagnosis of case 6 was G3 endometrioid carcinoma. While it is easy to overlook due to its rarity, diagnosis of undifferentiated/dedifferentiated carcinoma is essential, because it has a poorer prognosis than high-grade endometrioid carcinoma [20].

This study has a few limitations. First, the number of cases studied was small (a total of 21 cases) and the three reviewers produced diverse diagnoses, so there was no proper statistical method to calculate interobserver reproducibility. Second, only one of the three reviewers was an expert in gynecologic pathology. Since the two general pathologists were not familiar with the difficult histologic diagnosis of high-grade endometrial carcinoma, the interobserver discrepancy rate might have been measured higher than expected.

We investigated sequential changes in interobserver reproducibility in advanced-stage and high-grade endometrial carcinoma, with the stepwise addition of IHC results and molecular data. Accurate histological diagnosis of endometrial carcinoma subtypes is critical for patient management since several rare subtypes show unfavorable prognosis and require different treatment decisions. In conclusion, we demonstrated the utility of selected IHC markers and NGS molecular profiling in the diagnosis of advanced-stage endometrial carcinoma.

\section{Ethics Statement}

This study was approved by the Institutional Review Board of KUGH (ac- cession No. 2020GR0348). All the glass slides, paraffin blocks, and clinicopathological information were provided by the KUGH Biobank, which had collected patient samples and information with their informed consent.

\section{ORCID}

Ho Jin Jung https://orcid.org/0000-0002-5032-6178

Soo Yeon Lee https://orcid.org/0000-0003-0142-377X

Jin Hwa Hong https://orcid.org/0000-0002-6905-5363

Yi Kyeong Chun https://orcid.org/0000-0002-4235-9690

\section{Author Contributions}

Conceptualization: HJJ, SYL, JHH, YKC. Data curation: HJJ, SYL, YKC. Formal analysis: HJJ, SYL, YKC. Investigation: HJJ, SYL, YKC. Methodology: HJJ, JHH, YKC. Supervision: YKC. Validation: HJJ, SYL, JHH, YKC. Writing-original draft: HJJ, YKC. Writing_review \& editing: HJJ, SYL, YKC. Approval of final manuscript: all authors.

\section{Conflicts of Interest}

The authors declare that they have no potential conflicts of interest.

\section{Funding Statement}

No funding to declare.

\section{References}

1. Ferlay J, Soerjomataram I, Dikshit R, et al. Cancer incidence and mortality worldwide: sources, methods and major patterns in GLOBOCAN 2012. Int J Cancer 2015; 136: E359-86.

2. Lim MC, Won YJ, Ko MJ, et al. Incidence of cervical, endometrial, and ovarian cancer in Korea during 1999-2015. J Gynecol Oncol 2019; 30: e38.

3. Kurman RJ, Carcangiu ML, Herrington CS, Young RH. WHO classification of tumours of female reproductive organs. 4th ed. Lyon: IARC Press, 2014.

4. Bokhman JV. Two pathogenetic types of endometrial carcinoma. Gynecol Oncol 1983; 15: 10-7.

5. Wang J, Jia N, Li Q, et al. Analysis of recurrence and survival rates in grade 3 endometrioid endometrial carcinoma. Oncol Lett 2016; 12: $2860-7$.

6. Murali R, Davidson B, Fadare O, et al. High-grade endometrial carcinomas: morphologic and immunohistochemical features, diagnostic challenges and recommendations. Int J Gynecol Pathol 2019; 38 Suppl 1: S40-63.

7. Gilks CB, Oliva E, Soslow RA. Poor interobserver reproducibility in the diagnosis of high-grade endometrial carcinoma. Am J Surg Pathol 2013; 37: 874-81.

8. Han G, Sidhu D, Duggan MA, et al. Reproducibility of histological cell type in high-grade endometrial carcinoma. Mod Pathol 2013; 26: 1594-604.

9. Nastic D, Shanwell E, Wallin KL, et al. A selective biomarker panel increases the reproducibility and the accuracy in endometrial biopsy diagnosis. Int J Gynecol Pathol 2017; 36: 339-47.

10. Yen TT, Wang TL, Fader AN, Shih IM, Gaillard S. Molecular classification and emerging targeted therapy in endometrial cancer. Int J Gynecol Pathol 2020; 39: 26-35.

11. Hussein YR, Soslow RA. Molecular insights into the classification of high-grade endometrial carcinoma. Pathology 2018; 50: 151-61.

12. Kobel M, Ronnett BM, Singh N, Soslow RA, Gilks CB, McCluggage WG. Interpretation of P53 immunohistochemistry in endo- 
metrial carcinomas: toward increased reproducibility. Int J Gynecol Pathol 2019; 38 Suppl 1: S123-31.

13. Hoang LN, Kinloch MA, Leo JM, et al. Interobserver agreement in endometrial carcinoma histotype diagnosis varies depending on The Cancer Genome Atlas (TCGA)-based molecular subgroup. Am J Surg Pathol 2017; 41: 245-52.

14. Soslow RA, Tornos C, Park KJ, et al. Endometrial carcinoma diagnosis: use of FIGO grading and genomic subcategories in clinical practice: recommendations of the International Society of Gynecological Pathologists. Int J Gynecol Pathol 2019; 38 Suppl 1: S64-74.

15. Hoang LN, McConechy MK, Kobel M, et al. Histotype-genotype correlation in 36 high-grade endometrial carcinomas. Am J Surg Pathol 2013; 37: 1421-32.

16. Chen W, Husain A, Nelson GS, et al. Immunohistochemical profiling of endometrial serous carcinoma. Int J Gynecol Pathol 2017;
36: $128-39$.

17. Carlson JW, Nastic D. High-grade endometrial carcinomas: classification with molecular insights. Surg Pathol Clin 2019; 12: 343-62.

18. Horn LC, Hohn AK, Krucken I, Stiller M, Obeck U, Brambs CE. Mesonephric-like adenocarcinomas of the uterine corpus: report of a case series and review of the literature indicating poor prognosis for this subtype of endometrial adenocarcinoma. J Cancer Res Clin Oncol 2020; 146: 971-83.

19. Pocrnich CE, Ramalingam P, Euscher ED, Malpica A. Neuroendocrine carcinoma of the endometrium: a clinicopathologic study of 25 cases. Am J Surg Pathol 2016; 40: 577-86.

20. Yokomizo R, Yamada K, Iida Y, et al. Dedifferentiated endometrial carcinoma: a report of three cases and review of the literature. Mol Clin Oncol 2017; 7: 1008-12. 